\title{
Teleparallel Conformal Invariant Models Induced by Kaluza-Klein Reduction
}

\author{
Chao-Qiang Geng ${ }^{1,2,3,4, *}$ and Ling-Wei Luo ${ }^{2,+}$ \\ ${ }^{1}$ Chongqing University of Posts $\&$ Telecommunications, Chongqing, 400065, China \\ ${ }^{2}$ Department of Physics, National Tsing Hua University, Hsinchu 300, Taiwan \\ ${ }^{3}$ Physics Division, National Center for Theoretical Sciences, Hsinchu 300, Taiwan \\ ${ }^{4}$ Synergetic Innovation Center for Quantum Effects and Applications (SICQEA), \\ Hunan Normal University, Changsha 410081, China
}

\begin{abstract}
We study the extensions of teleparallism in the Kaluza-Klein (KK) scenario by writing the analogous form to the torsion scalar $T_{\mathrm{NGR}}$ in terms of the corresponding antisymmetric tensors, given by $T_{\mathrm{NGR}}=a T_{i j k} T^{i j k}+b T_{i j k} T^{k j i}+c T^{j}{ }_{j i} T^{k}{ }_{k}^{i}$, in the four-dimensional New General Relativity (NGR) with arbitrary coefficients $a, b$ and $c$. After the KK dimensional reduction, the Lagrangian in the Einstein-frame can be realized by taking $2 a+b+c=0$ with the ghost-free condition $c \leq 0$ for the one-parameter family of teleparallelism. We demonstrate that the pure conformal invariant gravity models can be constructed by the requirements of $2 a+b=0$ and $c=0$. In particular, the torsion vector can be identified as the conformal gauge field, while the conformal gauge theory can be obtained by $2 a+b+4 c=0$ or $2 a+b=0$, which is described on the Weyl-Cartan geometry $Y_{4}$ with the ghost-free conditions $2 a+b+c>0$ and $c \neq 0$. We also consider the weak field approximation and discuss the non-minimal coupled term of the scalar current and torsion vector. For the conformal invariant models with $2 a+b=0$, we find that only the anti-symmetric tensor field is allowed rather than the symmetric one.
\end{abstract}

\footnotetext{
* Electronic address: geng@phys.nthu.edu.tw

$\dagger$ Electronic address: lwluo@mx.nthu.edu.tw
} 


\section{INTRODUCTION}

The well-known five-dimension gravity theory is called the Kaluza-Klein (KK) theory [1, 2] with the concept of unifying electromagnetic and gravitational forces in the high dimensional spacetime. The fifth dimension is regarded as a circle $S^{1}$ under the spontaneous compactification, where the full spacetime manifold is a product manifold $M^{4} \times S^{1}$. The scalar-tensor theories can be induced by the KK reduction of the effective theories [3, 4].

The alternative gravitational theory under the absolutely parallelism was originally proposed by Einstein [5], in which the connection is constructed by the commutation coefficients and the Lagrangian would lead to the symmetric equations of motion [6]. Nowadays, we recognize that the geometry is the Weitzenböck geometry $T_{4}$ and the corresponding gravitational theory is called teleparallel equivalent to general relativity (TEGR). New General Relativity (NGR) was presented by Hayashi and Shirafuji [7], which is one of the generalizations in teleparallelism with the equivalence outcome as general relativity (GR). In NGR, the torsion scalar is given by

$$
T_{\mathrm{NGR}}=a T_{\rho \mu \nu} T^{\rho \mu \nu}+b T_{\rho \mu \nu} T^{\nu \mu \rho}+c T_{\nu \mu}^{\nu} T_{\sigma}^{\sigma}{ }^{\mu},
$$

where the torsion tensor is defined by the Weitzenböck connection and the coefficients $a$, $b$ and $c$ are arbitrary. In particular, the torsion scalar denoted by $T$ is given by $(a, b, c)=$ $(1 / 4,1 / 2,-1)$ for TEGR. The choices of the coefficients have been explored in the literature [7-12]. Recently, several specific extensions in teleparallelism have been investigated, such as models with the teleparallel dark energy [13] and $f(T)[14,15]$.

Teleparallelism in the KK scenario has been discussed in [16 21]. In this study, we consider the high dimensional gravity theory based on the analogous form to (1.1) in the four dimensional NGR theory. It is known that (1.1) in NGR provides a much richer structure than those in TEGR and GR due to the arbitrariness of the coefficients. Under the KK reduction, the effective Lagrangian will contain new couplings associated with some combinations of the coefficients. We will build the conformal invariant models in teleparallelism and examine the constraints on these new couplings

This paper is organized as follows. In Sec. II, we first introduce the NGR gravity theory on the Weitzenböck geometry in four dimensions and then extend it to a higher dimension. By following the KK reduction procedure, we obtain the effective Lagrangian in the fourdimensional spacetime. In Sec. III, we present some specific models with the conformal 
transformations for the zero modes in the effective Lagrangians. We study the conditions for having the theory in the Einstein-frame and with the conformal invariant, respectively. In Sec. IV, we analyze the weak field approximation of the four-dimensional effective theory and discuss the cases in the conformal invariant models. Finally, we give the conclusions in Sec. V.

\section{KALUZA-KLEIN REDUCTION IN TELEPARALLELISM}

\section{A. The Weitzenböck Background}

In the four-dimensional Weitzenböck geometry $T_{4}$, the orthonormal coframe $\vartheta^{i}=e_{\mu}^{i} d x^{\mu}$ is associated with the Minkowski metric $\eta_{i j}=\operatorname{diag}(+1,-1,-1,-1)$, which gives the relation of the four-dimensional metric $g_{\mu \nu}=\eta_{i j} e_{\mu}^{i} e_{\nu}^{j}$, where Greek indices $\mu, \nu, \rho, \ldots=0,1,2,3$ and Latin indices $i, j, k, \ldots=\hat{0}, \hat{1}, \hat{2}, \hat{3}$ denote as the coordinate and orthonormal frame indices, respectively 1 The frame fields (vielbein) $e_{\mu}^{i}$ and their inverse $e_{i}^{\mu}$ connect the coordinate and orthonormal frames via $e_{i}=e_{i}^{\mu} \partial_{\mu}$ and $\vartheta^{i}=e_{\mu}^{i} d x^{\mu}$, which satisfy the relations $e_{\mu}^{i} e_{j}^{\mu}=\delta_{j}^{i}$ and $e_{i}^{\mu} e_{\nu}^{i}=\delta_{\nu}^{\mu}$. In the Weitzenböck geometry, the absolute parallelism of the frame fields define the Weitzenböck connection from the zero covariant derivative of the frame fields $\nabla_{\nu} e_{\mu}^{i}=\partial_{\nu} e_{\mu}^{i}-e_{\rho}^{i} \Gamma_{\mu \nu}^{\rho}=0$, resulting in $\Gamma_{\mu \nu}^{\rho}=e_{i}^{\rho} \partial_{\nu} e_{\mu}^{i}$. The torsion tensor is given by the anti-symmetric part of the Weitzenböck connection $T^{\rho}{ }_{\mu \nu}=e_{i}^{\rho}\left(\partial_{\mu} e_{\nu}^{i}-\partial_{\nu} e_{\mu}^{i}\right)$. Consequently, the torsion scalar for TEGR can be written as the form in (1.1) in the coordinate frame, or

$$
T=a T_{i j k} T^{i j k}+b T_{i j k} T^{k j i}+c T_{j i}^{j} T_{k}^{k}
$$

in the orthonormal frame with $(a, b, c)=(1 / 4,1 / 2,-1)$. Subsequently, the torsion scalar can be rewritten as

$$
T=\frac{1}{2} T_{\mu \nu}^{\rho} S_{\rho}^{\mu \nu},
$$

where $S_{\rho}^{\mu \nu}=K_{\rho}^{\mu \nu}+\delta_{\rho}^{\mu} T_{\sigma}^{\sigma \nu}-\delta_{\rho}^{\nu} T_{\sigma}^{\sigma \mu}$ with the contorsion $K_{\mu \nu}^{\rho}=(-1 / 2)\left(T_{\mu \nu}^{\rho}-T_{\mu}{ }^{\rho}{ }_{\nu}-\right.$ $\left.T_{\nu}{ }^{\rho}{ }_{\mu}\right)$. The integration of the torsion scalar

$$
S_{\mathrm{TEGR}}=\frac{1}{2 \kappa} \int d^{4} x e T
$$

\footnotetext{
${ }^{1}$ We use the hatted number to represent the components in the orthonormal frame.
} 
yields the gravitational action equivalent to GR up to the divergence, so-called TEGR, where $\kappa=8 \pi G$ is the gravitational coupling and $e \equiv \operatorname{det}\left(e_{\mu}^{i}\right)$.

The generalization of TEGR can be realized by using the arbitrary coefficients $(a, b, c)$ instead of $(1 / 4,1 / 2,-1)$ in (1.1) and (2.1). In NGR, the Lagrangian density in the orthonormal frame is given by (2.1) without specific values for $(a, b, c)$, which can be reformulated to be

$$
T_{\mathrm{NGR}}=\frac{1}{2} T^{i}{ }_{j k} \Sigma_{i}{ }^{j k}
$$

where

$$
\Sigma_{i}^{j k}=2 a T_{i}{ }^{j k}+b\left(T^{k j}{ }_{i}-T^{j k}{ }_{i}\right)+c\left(\delta_{i}^{k} T^{l j}{ }_{l}-\delta_{i}^{j} T^{l k}{ }_{l}\right) .
$$

\section{B. The Kaluza-Klein Reduction}

\section{(i) Geometric part}

In the five-dimensional Weitzenböck geometry $T_{5}$, the metric is given by $\bar{g}_{M N}=\bar{\eta}_{I J} e_{M}^{I} e_{N}^{J}$, where $M, N=0,1,2,3,5, I, J=\hat{0}, \hat{1}, \hat{2}, \hat{3}, \hat{5}$, and $\bar{\eta}_{I J}=\operatorname{diag}(+1,-1,-1,-1,-1)$ is the metric in the orthonormal frame. In the orthonormal frame, it is convenient to calculate torsion components by the first Cartan structure equation $\bar{T}^{I}=d \bar{\vartheta}^{I}$. The decompositions of the torsion 2-forms are $\bar{T}^{i}=(1 / 2) \bar{T}_{j k}^{i} \bar{\vartheta}^{j} \wedge \bar{\vartheta}^{k}+\bar{T}^{i}{ }_{\hat{5} j} \bar{\vartheta}^{\hat{5}} \wedge \bar{\vartheta}^{j}$ and $\bar{T}^{\hat{5}}=(1 / 2) \bar{T}^{\hat{5}_{j j}} \bar{\vartheta}^{i} \wedge \bar{\vartheta}^{j}+$ $\bar{T}_{\hat{5} i} \bar{\vartheta}^{\hat{5}} \wedge \bar{\vartheta}^{i}$.

In the Kaluza-Klein theory, we take the five-dimensional spacetime to be a product manifold $T_{5}=T_{4} \times S^{1}$ with the fifth dimension compactifying to $S^{1}$ and the five-dimensional metric $\bar{g}$ in the coordinate frame as

$$
\bar{g}_{M N}=\left(\begin{array}{cc}
g_{\mu \nu}-\kappa^{2} \phi^{2} A_{\mu} A_{\nu} & \kappa \phi^{2} A_{\mu} \\
\kappa \phi^{2} A_{\nu} & -\phi^{2}
\end{array}\right),
$$

where $\bar{g}_{\mu \nu}, A_{\mu}$ and $\phi$ depend on $x^{\mu}$ and $y$ coordinates, and the constant $\kappa$ has a mass dimension of $[\kappa]=-1$. The corresponding coframes in $T_{5}$ are

$$
\begin{aligned}
& \bar{\vartheta}^{i}=e_{\mu}^{i} d x^{\mu}, \\
& \bar{\vartheta}^{\hat{5}}=e_{\mu}^{\hat{5}} d x^{\mu}+e_{5}^{\hat{5}} d x^{5}=-\kappa \phi A_{\mu} d x^{\mu}+\phi d y
\end{aligned}
$$

with $y \equiv x^{5}$. From (2.7a) and (2.7b), we have the inverse relation

$$
d y=e_{i}^{5} \bar{\vartheta}^{i}+e_{\hat{5}}^{5} \bar{\vartheta}^{\hat{5}}=\kappa A_{\mu} e_{i}^{\mu} \bar{\vartheta}^{i}+\frac{1}{\phi} \bar{\vartheta}^{\hat{5}} .
$$


The components of the torsion tensor in the orthonormal frame are obtained by

$$
\begin{aligned}
\bar{T}_{j k}^{i} & =T^{i}{ }_{j k}+\kappa A_{\mu}\left(\partial_{5} e_{\nu}^{i}\right)\left(e_{j}^{\mu} e_{k}^{\nu}-e_{k}^{\mu} e_{j}^{\nu}\right), \\
\bar{T}^{i}{ }_{\hat{5}_{j}} & =\frac{1}{\phi}\left(\partial_{5} e_{\mu}^{i}\right) e_{j}^{\mu}, \\
\bar{T}^{\hat{5}_{i j}} & =-\frac{\kappa}{2} \phi e_{i}^{\mu} e_{j}^{\nu} F_{\mu \nu}+\kappa^{2} \phi A_{\mu}\left(\partial_{5} A_{\nu}\right)\left(e_{i}^{\nu} e_{j}^{\mu}-e_{j}^{\nu} e_{i}^{\mu}\right), \\
\bar{T}^{\hat{5}}{ }_{i \hat{5}} & =\frac{1}{\phi} e_{i}^{\mu}\left(\partial_{\mu} \phi\right)+\frac{1}{\phi} \kappa A_{\mu} e_{i}^{\mu}\left(\partial_{5} \phi\right)+\kappa e_{i}^{\mu}\left(\partial_{5} A_{\mu}\right),
\end{aligned}
$$

where $F_{\mu \nu}:=\partial_{\mu} A_{\nu}-\partial_{\nu} A_{\mu}$ and $T^{i}{ }_{j k}=e_{j}^{\mu}\left(\partial_{\mu} e_{\nu}^{i}\right) e_{k}^{\nu}-e_{k}^{\mu}\left(\partial_{\mu} e_{\nu}^{i}\right) e_{j}^{\nu}$, which are four-dimensional quantities without involving the fifth component.

The extended torsion scalar in $D$-dimension, ${ }^{(D)} T^{(\mathrm{ext})}$, can be shown in a similar way as (2.4). In particular, the five-dimensional extended torsion scalar is given by

$$
{ }^{(5)} T^{(\mathrm{ext})}=a \bar{T}_{L M N} \bar{T}^{L M N}+b \bar{T}_{L M N} \bar{T}^{N M L}+c \bar{T}^{L}{ }_{L M} \bar{T}^{N}{ }_{N}{ }^{M} .
$$

We decompose the contraction of the fifth component part from the extended torsion scalar, expressed as

$$
\begin{aligned}
{ }^{(5)} T^{(\mathrm{ext})}= & \bar{T}_{\mathrm{NGR}}+2 a \bar{T}_{i \hat{i} j} \bar{T}^{i \hat{5} j}+a \bar{T}_{\hat{5} i j} \bar{T}^{5 i j}+b \bar{T}_{i \hat{5} j} \bar{T}^{j \hat{5} i}+2 b \bar{T}_{\hat{5} i j} \bar{T}^{j i \hat{5}} \\
& +(2 a+b+c) \bar{T}_{\hat{5} i \hat{5}} \bar{T}^{\hat{5} i \hat{5}}+2 c \bar{T}^{j}{ }_{j}{ }_{j} \bar{T}_{{ }_{\hat{5}}{ }^{i}+c \bar{T}^{i}{ }_{i \hat{5}} \bar{T}^{j}{ }_{j}{ }^{5}}
\end{aligned}
$$

where the torsion scalar $\bar{T}_{\mathrm{NGR}}$ is in the five-dimensional geometric form, constructed by $\bar{T}^{i}{ }_{j k}$ in (2.9a). According to (2.9), we can write down 8 terms for each extended five-dimensional torsion scalar (2.11), as shown in Appendix A.

One can consider the $n$-mode harmonic expansions with the fifth dimensional radius $r$ for $e_{\mu}^{i}, A_{\mu}$ and $\phi$, given by

$$
\begin{aligned}
e_{\mu}^{i}\left(x^{\mu}, y\right) & =\sum_{n} e_{\mu}^{i(n)}\left(x^{\mu}\right) e^{i n y / 2 r}, \\
A_{\mu}\left(x^{\mu}, y\right) & =\sum_{n} A_{\mu}^{(n)}\left(x^{\mu}\right) e^{i n y / r} \\
\phi\left(x^{\mu}, y\right) & =\sum_{n} \phi^{(n)}\left(x^{\mu}\right) e^{i n y / r}
\end{aligned}
$$

respectively. We specify the physical gravitational fields as the metric tensor with the harmonic expansion of the form $g_{\mu \nu}(x, y)=\sum_{n} g_{\mu \nu}^{(n)}(x) e^{i n y / r}$. Similarly, the harmonic expansion of $e_{i}^{\mu}$ is defined by $e_{i}^{\mu}(x, y)=\sum_{n} e_{i}^{\mu(n)}(x) e^{-i n y / 2 r}$ in order to satisfy the orthonormality relation. The mass squared $n^{2} / r^{2}$ will be generated by the derivative respect to the $y$ coordinate. 
The action of the zeroth KK mode $(n=0)$ in the four-dimensional effective extended gravity in teleparallism can be written as

$$
\begin{aligned}
S_{g}^{(0)}= & \int d^{4} x e^{(0)}\left(\frac{1}{2 \kappa_{4}} \phi^{(0)} T_{\mathrm{NGR}}^{(0)}-\frac{a \kappa^{2}}{8 \kappa_{4}} \phi^{(0) 3} g^{(0) \mu \rho} g^{(0) \nu \sigma} F_{\mu \nu}^{(0)} F_{\rho \sigma}^{(0)}\right. \\
& \left.+\frac{2 a+b+c}{2 \kappa_{4}} \frac{1}{\phi^{(0)}} g^{(0) \mu \nu} \partial_{\mu} \phi^{(0)} \partial_{\nu} \phi^{(0)}-\frac{c}{\kappa_{4}} g^{(0) \mu \nu} T_{\mu}^{(0)} \partial_{\nu} \phi^{(0)}\right),
\end{aligned}
$$

where $\kappa_{4}:=\kappa_{5} / \lambda=8 \pi G_{5} / \lambda$ is the effective gravitational constant with the fifth dimensional integral factor $\lambda \equiv 2 \pi r, \kappa$ can be chosen as $\sqrt{\kappa_{4}}$, and $T_{\mu}^{(0)}:=T^{(0) \nu}{ }_{\nu \mu}$ is the torsion vector. The effective action (2.13) is invariant under the global conformal transformation in the absence of the vector field $A_{\mu}^{(0)}$, which can be identified as the electromagnetic field, while the associated Noether current density is $J_{\mu}^{(0)}:=e(2 a+b+c) \partial_{\mu} \phi^{(0)} / \kappa_{4}$.

Note that in the case of the five-dimensional TEGR theory, the action (2.13) with $(a, b, c)=(1 / 4,1 / 2,-1)$ can be reduced to those in [16] and [20, 21] with $\phi^{(0)}=1$ and $A_{\mu}^{(0)}=0$, respectively.

\section{(ii) Matter part}

We now add the matter Lagrangian ${ }^{(5)} \mathcal{L}_{\mathrm{m}}={ }^{(5)} e L_{\mathrm{m}}\left(e_{M}^{I}, \Psi, \mathcal{D}_{M} \Psi\right)$ into the effective action (2.13), where $\mathcal{D}_{M}$ is the covariant derivative of the matter field $\Psi$. The $n$-mode harmonic expansion of $\Psi$ is given by

$$
\Psi\left(x^{\mu}, y\right)=\sum_{n} \Psi\left(x^{\mu}\right) e^{i n y / r} .
$$

The term $\mathcal{D}_{5} \Psi$ will produce the KK mass terms for the $\Psi$ field. For the massless zero mode, the matter field $\Psi^{(0)}$ is assumed to be localized on the $T_{4}$ hypersurface. After the KK reduction, the effective matter Lagrangian becomes

$$
\mathcal{L}_{\mathrm{m}, \mathrm{eff}}^{(0)}=e^{(0)} \lambda \phi^{(0)} L_{\mathrm{m}}\left(e_{\mu}^{i(0)}, A_{\mu}^{(0)}, \phi^{(0)}, \Psi^{(0)}, \mathcal{D}_{\mu} \Psi^{(0)}\right)
$$

Without confusion, we shall drop all the superscript (0) in our discussion for the fourdimensional effective theory. We can vary the full action $S=S_{g}+S_{\mathrm{m}}$ with respect to $e_{\mu}^{i}$, $A_{\mu}$ and $\phi$, resulting in

$$
\begin{array}{r}
\frac{1}{2} e_{i}^{\mu}\left(\phi T_{\mathrm{NGR}}-\frac{a \kappa^{2}}{4} \phi^{3} g^{\lambda \rho} g^{\nu \sigma} F_{\lambda \nu} F_{\rho \sigma}+\frac{2 a+b+c}{\phi} g^{\lambda \nu} \partial_{\lambda} \phi \partial_{\nu} \phi\right. \\
\left.-2 c g^{\lambda \nu} T_{\lambda} \partial_{\nu} \phi\right)-e_{i}^{\rho}\left\{\phi T_{\rho \nu}^{j} \Sigma_{j}{ }^{\mu \nu}-\frac{a \kappa^{2}}{2} \phi^{3} g^{\mu \lambda} g^{\nu \sigma} F_{\lambda \nu} F_{\rho \sigma}\right. \\
\left.+\frac{2 a+b+c}{\phi} g^{\mu \lambda} \partial_{\lambda} \phi \partial_{\rho} \phi-c\left(\partial_{\sigma} \phi T_{\rho}^{\mu}{ }^{\sigma}+\partial_{\rho} \phi T^{\mu}+\partial^{\mu} \phi T_{\rho}\right)\right\}
\end{array}
$$




$$
\begin{gathered}
+\frac{1}{e} \partial_{\nu}\left\{e\left(\phi \Sigma_{i}{ }^{\mu \nu}-c e_{i}^{\mu} \partial^{\nu} \phi+c e_{i}^{\nu} \partial^{\mu} \phi\right)\right\}=\kappa_{4} \lambda \phi \Theta_{i}^{\mu}, \\
\frac{\lambda}{e} \frac{\delta \mathcal{L}_{\mathrm{m}}}{\delta A_{\nu}}+\frac{1}{e} \frac{a \kappa^{2}}{2 \kappa_{4}} \phi^{2} \partial_{\mu}\left(e F^{\mu \nu}\right)+\frac{3 a \kappa^{2}}{2 \kappa_{4}} \phi \partial_{\mu} \phi F^{\mu \nu}=0 \\
T_{\mathrm{NGR}}-\frac{3 a \kappa^{2}}{4} \phi^{2} g^{\mu \rho} g^{\nu \sigma} F_{\mu \nu} F_{\rho \sigma}+2 \kappa_{4} \lambda\left(L_{\mathrm{m}}+\frac{\phi}{e} \frac{\delta \mathcal{L}_{\mathrm{m}}}{\delta \phi}\right) \\
+\frac{2 a+b+c}{\phi^{2}}\left(g^{\mu \nu} \partial_{\mu} \phi \partial_{\nu} \phi-2 \phi \hat{\square} \phi\right)+\frac{2 c}{e} \partial_{\nu}\left(e g^{\mu \nu} T_{\mu}\right)=0,
\end{gathered}
$$

respectively, where $\hat{\square}:=(1 / e) \partial_{\nu}\left(e g^{\mu \nu} \partial_{\mu}\right)$ and $\Theta_{i}^{\mu}:=(-1 / e)\left(\delta \mathcal{L}_{m} / \delta e_{\mu}^{i}\right)$ is the dynamical energy-momentum tensor with $\mathcal{L}_{\mathrm{m}}:=e L_{\mathrm{m}}$.

\section{SPECIFIC MODELS}

We now concentrate on the four-dimensional effective theory in (2.13). With the conformal transformations of the metric tensor and frame fields to be $\widetilde{g}_{\mu \nu}=\Omega^{2}(x) g_{\mu \nu}$ and $\widetilde{e}_{\mu}^{i}=\Omega e_{\mu}^{i}$, respectively, the corresponding matter Lagrangian is given by

$$
\lambda \phi \mathcal{L}_{\mathrm{m}}\left(g^{\mu \nu}, A_{\mu}, \phi, \Psi, \mathcal{D}_{\mu} \Psi\right)=\lambda \phi \Omega^{-4} \widetilde{e} L_{\mathrm{m}}\left(\Omega^{2} \widetilde{g}^{\mu \nu}, \widetilde{A}_{\mu}, \widetilde{\phi}, \widetilde{\Psi}, \widetilde{\mathcal{D}}_{\mu} \widetilde{\Psi}\right)
$$

where $e=\Omega^{-4} \widetilde{e}$. In the 4-dimensional spacetime, we have the conformal transformations

$$
\begin{aligned}
\widetilde{T}_{\mu \nu}^{i} & =\Omega T_{\mu \nu}^{i}+\left(\partial_{\mu} \Omega\right) e_{\nu}^{i}-e_{\mu}^{i}\left(\partial_{\nu} \Omega\right), \\
\widetilde{T}_{\mu} & =T_{\mu}-3 \Omega^{-1} \partial_{\mu} \Omega
\end{aligned}
$$

for the torsion tensor and vector, respectively. By extending the transformations to the torsion scalar of NGR, we obtain

$$
\begin{aligned}
T_{\mathrm{NGR}}= & \Omega^{2} \widetilde{T}_{\mathrm{NGR}}+(4 a+2 b+6 c) \widetilde{g}^{\mu \nu} \widetilde{T}_{\mu}\left(\Omega \partial_{\nu} \Omega\right) \\
& +(6 a+3 b+9 c) \widetilde{g}^{\mu \nu} \partial_{\mu} \Omega \partial_{\nu} \Omega
\end{aligned}
$$

In Appendix B, we show the formulas of the $D$-dimensional conformal transformation for the torsion tensor.

For the massless scalar field $\phi$, the conformal transformation is $\widetilde{\phi}=\Omega^{\lambda_{\phi}} \phi$ with the conformal weight parameter $\lambda_{\phi}$. On the other hand, one has $\widetilde{A}_{\mu}=A_{\mu}$ and $\widetilde{F}_{\mu \nu}=F_{\mu \nu}$ for the vector field and field strength, which are independent of the metric, respectively. Finally, 
the conformal transformed effective Lagrangian is found to be

$$
\begin{aligned}
& \mathcal{L}_{g}=\widetilde{e} \frac{\Omega^{-\left(\lambda_{\phi}+2\right)}}{2 \kappa_{4}}\left\{\widetilde{\phi} \widetilde{T}_{\mathrm{NGR}}+\Omega^{-1}\left(4 a+2 b+\left(6+2 \lambda_{\phi}\right) c\right) \widetilde{\phi} \widetilde{g}^{\mu \nu} \widetilde{T}_{\mu} \partial_{\nu} \Omega\right. \\
&+\Omega^{-2}\left(\left(6+2 \lambda_{\phi}^{2}\right) a+\left(3+\lambda_{\phi}^{2}\right) b+\left(9+6 \lambda_{\phi}+\lambda_{\phi}^{2}\right) c\right) \widetilde{\phi} \widetilde{g}^{\mu \nu} \partial_{\mu} \Omega \partial_{\nu} \Omega \\
&-\Omega^{-2\left(\lambda_{\phi}-1\right)} \frac{a \kappa^{2}}{4} \widetilde{\phi}^{3} \widetilde{g}^{\mu \rho} \widetilde{g}^{\nu \sigma} \widetilde{F}_{\mu \nu} \widetilde{F}_{\rho \sigma}+(2 a+b+c) \frac{1}{\widetilde{\phi}} \widetilde{g}^{\mu \nu} \partial_{\mu} \widetilde{\phi} \partial_{\nu} \widetilde{\phi} \\
&\left.-2 c \widetilde{g}^{\mu \nu} \widetilde{T}_{\mu} \partial_{\nu} \widetilde{\phi}+\Omega^{-1}\left(-4 \lambda_{\phi} a-2 \lambda_{\phi} b-\left(2 \lambda_{\phi}+6\right) c\right) \widetilde{g}^{\mu \nu} \partial_{\mu} \Omega \partial_{\nu} \widetilde{\phi}\right\} .
\end{aligned}
$$

Due to the undetermined coefficients $(a, b, c)$, we would examine the necessary conditions for having the Einstein-frame as well as conformal invariant models. By taking $\lambda_{\phi}=-2$ with the conformal scalar $\omega:=\ln \Omega$, the Lagrangian (3.4) becomes

$$
\begin{aligned}
& \mathcal{L}_{g}=\widetilde{e} \frac{1}{2 \kappa_{4}}\left\{\widetilde{\phi} \widetilde{T}_{\mathrm{NGR}}+(4 a+2 b+2 c) \widetilde{\phi} \widetilde{g}^{\mu \nu} \widetilde{T}_{\mu} \partial_{\nu} \omega\right. \\
&+(14 a+7 b+c) \widetilde{\phi} \widetilde{g}^{\mu \nu} \partial_{\mu} \omega \partial_{\nu} \omega \\
&-e^{6 \omega} \frac{a \kappa^{2}}{4} \widetilde{\phi}^{3} \widetilde{g}^{\mu \rho} \widetilde{g}^{\nu \sigma} \widetilde{F}_{\mu \nu} \widetilde{F}_{\rho \sigma}+(2 a+b+c) \frac{1}{\widetilde{\phi}} \widetilde{g}^{\mu \nu} \partial_{\mu} \widetilde{\phi} \partial_{\nu} \widetilde{\phi} \\
&\left.-2 c \widetilde{g}^{\mu \nu} \widetilde{T}_{\mu} \partial_{\nu} \widetilde{\phi}+(8 a+4 b-2 c) \widetilde{g}^{\mu \nu} \partial_{\mu} \omega \partial_{\nu} \widetilde{\phi}\right\} .
\end{aligned}
$$

\section{A. Einstein-Frame Condition}

In general, the Einstein-frame does not exist for the non-minimal torsion scalar $\phi T_{\mathrm{NGR}}$ due to the non-minimal coupling $\widetilde{g}^{\mu \nu} \widetilde{T}_{\mu}\left(\Omega \partial_{\nu} \Omega\right)$ generated in (3.3), which is different from the transformation of the curvature scalar in GR. In order to transform the effective action (2.13) from the Jordan-frame to Einstein-frame, we only focus on the gravitational part and choose $\widetilde{\phi}=\Omega^{-2} \phi=1$. If the coefficients in (3.5) satisfy the condition

$$
2 a+b+c=0,
$$

the Einstein-frame is obtained by eliminating the term $\widetilde{g}^{\mu \nu} \widetilde{T}_{\mu} \partial_{\nu} \omega$. As a result, the Lagrangian in $(2.13)$ is reduced to

$$
\mathcal{L}_{g}=e \frac{1}{2 \kappa_{4}}\left(\phi T_{\mathrm{NGR}}-2 c g^{\mu \nu} T_{\mu} \partial_{\nu} \phi-\frac{a \kappa^{2}}{4} \phi^{3} g^{\mu \rho} g^{\nu \sigma} F_{\mu \nu} F_{\rho \sigma}\right) .
$$


In the corresponding Einstein-frame, it reads as

$$
\mathcal{L}_{g}^{(\mathrm{E})}=\widetilde{e}\left(\frac{1}{2 \kappa_{4}} \widetilde{T}_{\mathrm{NGR}}-\frac{c}{2} \widetilde{g}^{\mu \nu} \partial_{\mu} \varphi \partial_{\nu} \varphi-\frac{a \kappa^{2}}{8 \kappa_{4}} e^{6 \omega} \widetilde{g}^{\mu \rho} \widetilde{g}^{\nu \sigma} \widetilde{F}_{\mu \nu} \widetilde{F}_{\rho \sigma}\right),
$$

where $\varphi:=\sqrt{6 / \kappa_{4}} \omega$, As seen from (3.8), to avoid the ghost field, one has an additional condition $c \leq 0$. As a result, the conditions

$$
2 a+b+c=0 \quad \text { and } \quad c \leq 0
$$

are needed to have the Einstein-frame. For a simple choice of $c=-1$, one gets the minimal coupled one-parameter family model with $2 a+b=1$ in teleparallelism [7, 10, 11].

We note that the coefficients $(a, b, c)=(1 / 4,1 / 2,-1)$ satisfy (3.6), which reduce (3.7) to be the case of TEGR in the KK theory with the gauge field $A_{\mu}$, whereas that for the Lagrangian (3.8) of TEGR in the Einstein-frame without $A_{\mu}$ has been presented in [21].

\section{B. Conformal Invariant}

It is apparent that (3.4) or (3.5) is not conformal invariant in the presence of the vector field $A_{\mu}$. For the case without $A_{\mu}$, from (3.5) we obtain the requirements of a conformal invariant theory by only keeping the terms of $\widetilde{\phi}^{-1} \widetilde{g}^{\mu \nu} \partial_{\mu} \widetilde{\phi} \partial_{\nu} \widetilde{\phi}$ and $\widetilde{g}^{\mu \nu} \widetilde{T}_{\mu} \partial_{\nu} \widetilde{\phi}$, given by

$$
\begin{aligned}
& 4 a+2 b+2 c=0, \\
& 14 a+7 b+c=0, \\
& 8 a+4 b-2 c=0 .
\end{aligned}
$$

The solution for (3.10) is

$$
2 a+b=0 \quad \text { and } \quad c=0
$$

corresponding to the simple one-parameter conformal invariant gravity in teleparallelism. The action is given by

$$
S_{g}^{(\mathrm{c})}=\int d^{4} x\left\{e \frac{a}{2 \kappa_{4}} \phi\left(T_{i j k} T^{i j k}-2 T_{i j k} T^{k j i}\right)+\lambda \phi \mathcal{L}_{\mathrm{m}}\right\},
$$

where $\phi$ is the auxiliary field, which can be identified as the varying Newton's constant. For the variation with respect to the frame fields $e_{\mu}^{i}$, we get the gravitational equation of motion.

$$
\begin{aligned}
& \phi\left\{\frac{1}{2} e_{i}^{\mu} T_{\rho \nu}^{j}\left(T_{j}{ }^{\rho \nu}-2 T^{\nu \rho}{ }_{j}\right)-4 e_{i}^{\rho} T^{j}{ }_{\rho \nu} K^{\mu \nu}{ }_{j}\right\} \\
& +\frac{2 \phi}{e} \partial_{\nu}\left\{e\left(T_{i}{ }^{\mu \nu}-2 T^{\nu \mu}{ }_{i}\right)\right\}+2\left(T_{i}^{\mu \nu}-2 T^{\nu \mu}{ }_{i}\right) \partial_{\nu} \phi=\kappa_{4} \lambda \phi \Theta_{i}^{\mu} .
\end{aligned}
$$


Furthermore, the equation of motion of $\phi$ leads to the constraint

$$
a T_{i j k}\left(T^{i j k}-2 T^{k j i}\right)+2 \kappa_{4} \lambda\left(L_{\mathrm{m}}+\frac{\phi}{e} \frac{\delta \mathcal{L}_{\mathrm{m}}}{\delta \phi}\right)=0 .
$$

In a pure gravity theory, (3.14) yields (i) $T_{i j k}=0$ or (ii) $T^{i j k}=2 T^{k j i}$. Note that (i) is the torsion-free case, which is forbidden due to no gravity. For (ii), it implies that $T^{i i k}=2 T^{k i i}=$ 0 , which contains no torsion vector mode, so that the equation of motion (3.13) reads

$$
e_{i}^{\rho} T_{\rho \nu}^{j} T_{j}^{\nu \mu}=0 \text {. }
$$

On the other hand, if we assume that $\omega=\omega(\phi)$, under the conformal transformation, $\widetilde{\phi}$ could be expressed as the function $\widetilde{\phi}(\phi)$, as long as $\operatorname{det}(d \widetilde{\phi} / d \phi)$ exists. Subsequently, we can have $\phi(\widetilde{\phi})$ by the inverse function theorem, resulting in $\omega(\widetilde{\phi})$ by $d \widetilde{\phi} / d \phi=\omega^{\prime} \exp \left(\lambda_{\phi} \omega\right)(1+$ $\left.\lambda_{\phi} \omega^{\prime} \phi\right)$ with $\omega^{\prime}:=d \omega / d \phi$. By setting

$$
\partial_{\mu} \omega=\partial_{\mu} \ln \widetilde{\phi}
$$

the Lagrangian density (3.5) without $A_{\mu}$ reads

$$
\mathcal{L}_{g}=\widetilde{e} \frac{1}{2 \kappa_{4}}\left\{\widetilde{\phi} \widetilde{T}_{\mathrm{NGR}}+(4 a+2 b) \widetilde{g}^{\mu \nu} \widetilde{T}_{\mu} \partial_{\nu} \widetilde{\phi}+(24 a+12 b) \frac{1}{\widetilde{\phi}} \widetilde{g}^{\mu \nu} \partial_{\mu} \widetilde{\phi} \partial_{\nu} \widetilde{\phi}\right\} .
$$

By comparing the coefficients in (2.13), the new requirements of the conformal invariance are found to be

$$
\begin{aligned}
2 a+b & =-c, \\
24 a+12 b & =2 a+b+c,
\end{aligned}
$$

leading to the solution of $2 a+b=0$ and $c=0$ as (3.11). It is apparent that the conformal invariance gives rise to $2 a+b+c=0$, which is the same as (3.6) for the existence of the Einstein-frame.

\section{Weyl Gauge Invariant}

In this subsection, we would like to discuss the gauge theory in which the gauge field would be introduced from the covariant derivative of the scalar filed $\phi$. According to (2.13) without the $A_{\mu}$ field, the Lagrangian can be rewritten as

$$
\begin{aligned}
\mathcal{L}_{g}=e \frac{1}{2 \kappa_{4}}\left\{\phi\left(T_{\mathrm{NGR}}-k c g^{\mu \nu} T_{\mu} T_{\nu}\right)\right. & \\
& \left.+\frac{c}{k \phi}\left(g^{\mu \nu}\left(\partial_{\mu}-k T_{\mu}\right) \phi\left(\partial_{\nu}-k T_{\nu}\right) \phi\right)\right\},
\end{aligned}
$$


where $k=c /(2 a+b+c)$ is a fixed ratio with $2 a+b+c \neq 0$ and $c \neq 0$, satisfying the equation

$$
\frac{1}{k}+2-3 k=0
$$

as shown in Appendix C]. We remark that $c / k=2 a+b+c>0$ is the ghost-free condition for this model. As a result, we obtain the conformal gauge invariant condition to be either

$$
2 a+b+4 c=0
$$

or

$$
2 a+b=0
$$

with the constraints

$$
2 a+b+c>0 \quad \text { and } \quad c \neq 0 .
$$

The torsion vector $T_{\mu}$ in (3.19) can be identified as the gauge field under the gauge principle for the conformal/Weyl transformation. Under the transformations

$$
g_{\mu \nu} \longrightarrow e^{2 \omega} g_{\mu \nu}, \quad T_{\mu} \longrightarrow T_{\mu}-3 \partial_{\mu} \omega, \quad \phi \longrightarrow e^{-2 \omega} \phi
$$

the modified derivative of the $\psi$ field can be defined by ${ }^{*} \partial_{\mu}^{(\psi)}=\partial_{\mu}+\left(\lambda_{\psi} k / 2\right) T_{\mu}$, where the weight parameter $\lambda_{\psi}$ is defined by the transformation $\exp \left(\lambda_{\psi} \omega\right)$. In addition, we have $\lambda_{\phi}=-2$ for $\phi$. It can be shown that ${ }^{*} \partial_{\mu}^{(e)} e_{\nu}^{i}=\partial_{\mu} e_{\nu}^{i}+(k / 2) T_{\mu} e_{\nu}^{i}$ due to $\lambda_{g}=2 \lambda_{e}=2$. We can define a modified covariant derivative for $\psi$ given by ${ }^{*} \nabla^{(\psi)}={ }^{*} d^{(\psi)}+{ }^{*} \Gamma$ with the connection ${ }^{*} \Gamma_{\nu \mu}^{\rho}=e_{i}^{\rho *} \partial_{\mu}^{(e)} e_{\nu}^{i}=\Gamma_{\nu \mu}^{\rho}+(k / 2) \delta_{\nu}^{\rho} T_{\mu}$. Under this new covariant derivative, the nonmetricity vanishes, i.e.,

$$
{ }^{*} \nabla_{\mu}^{(g)} g_{\nu \rho}={ }^{*} \partial_{\mu}^{(g)} g_{\nu \rho}-{ }^{*} \Gamma_{\nu \mu}^{\sigma} g_{\sigma \rho}-{ }^{*} \Gamma_{\rho \mu}^{\sigma} g_{\nu \sigma}=\nabla_{\mu} g_{\nu \rho}=0
$$

Furthermore, we have

$$
\begin{aligned}
{ }^{*} T_{\mu \nu}^{\rho} & =T_{\mu \nu}^{\rho}+\frac{k}{2}\left(\delta_{\nu}^{\rho} T_{\mu}-\delta_{\mu}^{\rho} T_{\nu}\right), \\
{ }^{*} T_{\mu} & =\left(1-\frac{3}{2} k\right) T_{\mu},
\end{aligned}
$$

and we can define ${ }^{*} \widetilde{T}^{\rho}{ }_{\mu \nu}:=\widetilde{\left({ }^{*} T\right)^{\rho}}{ }_{\mu \nu}$, read as

$$
\begin{aligned}
{ }^{*} \widetilde{T}_{\mu \nu}^{\rho} & =\widetilde{T}_{\mu \nu}^{\rho}+\frac{k}{2}\left(\delta_{\nu}^{\rho} \widetilde{T}_{\mu}-\delta_{\mu}^{\rho} \widetilde{T}_{\nu}\right), \\
{ }^{*} \widetilde{T}_{\mu} & =\left(1-\frac{3}{2} k\right) \widetilde{T}_{\mu} .
\end{aligned}
$$


Note that $\widetilde{\left({ }^{*} T\right)^{\rho}}{ }_{\mu \nu} \neq *(\widetilde{T})^{\rho}{ }_{\mu \nu}$. We can rewrite the derivative as ${ }^{*} \partial_{\mu}^{(\phi)}:=\partial_{\mu}-\lambda_{\phi} k^{2 *} T_{\mu}$ in (3.19) from (3.20) and (3.26b), which leads to the modified covariant derivative ${ }^{*} \nabla_{\mu}^{(\phi)} \phi=$ ${ }^{*} \partial_{\mu}^{(\phi)} \phi$ with the new connection ${ }^{*} \Gamma_{\nu \mu}^{\rho}$. Under the conformal transformation, the nonmetricity with respect to the modified connection is

$$
{ }^{*} \widetilde{\nabla}_{\mu}^{(g)} \widetilde{g}_{\nu \rho}=e^{2 \omega}\left({ }^{*} \nabla_{\mu}^{(g)} g_{\nu \rho}\right)=0,
$$

which implies that ${ }^{*} \widetilde{\nabla}_{\mu}^{(g)}$ is an invariant covariant derivative. With the condition (3.21) or (3.22), we have

$$
\begin{aligned}
& e \phi\left(T_{\mathrm{NGR}}-k c g^{\mu \nu} T_{\mu} T_{\nu}\right) \\
= & e \phi\left({ }^{*} T_{\mathrm{NGR}}-k c g^{\mu \nu *} T_{\mu}{ }^{*} T_{\nu}\right) \\
= & \widetilde{e} \widetilde{\phi}\left({ }^{*} \widetilde{T}_{\mathrm{NGR}}-k c \widetilde{g}^{\mu \nu *} \widetilde{T}_{\mu}{ }^{*} \widetilde{T}_{\nu}\right),
\end{aligned}
$$

where we have used the relation ${ }^{*} \widetilde{T}^{\rho}{ }_{\mu \nu}={ }^{*} T^{\rho}{ }_{\mu \nu}-(1 / 2)\left(\delta_{\nu}^{\rho} \partial_{\mu} \omega-\delta_{\mu}^{\rho} \partial_{\nu} \omega\right)$. Consequently, (3.19) becomes

$$
\mathcal{L}_{g}=\widetilde{e} \frac{1}{2 \kappa_{4}}\left\{\widetilde{\phi}\left({ }^{*} \widetilde{T}_{\mathrm{NGR}}-k c g^{\mu \nu} * \widetilde{T}_{\mu}{ }^{*} \widetilde{T}_{\nu}\right)+\frac{c}{k \widetilde{\phi}} \widetilde{g}^{\mu \nu *} \widetilde{\nabla}_{\mu}^{(\phi)} \widetilde{\phi}^{*} \widetilde{\nabla}_{\nu}^{(\phi)} \widetilde{\phi}\right\}
$$

where the covariant derivative for $\widetilde{\phi}$ is given by

$$
{ }^{*} \widetilde{\nabla}_{\mu}^{(\phi)}=* \widetilde{\partial}_{\mu}^{(\phi)}+\left(\frac{3}{2} \lambda_{\phi} k^{2}-\lambda_{\phi}\right) \partial_{\mu} \omega
$$

with $* \widetilde{\partial}_{\mu}^{(\phi)}=\partial_{\mu}-\lambda_{\phi} k^{2} * \widetilde{T}_{\mu}$. which has the same form as $\phi$ under the transformation, given by ${ }^{*} \widetilde{\nabla}_{\mu}^{(\phi)} \widetilde{\phi}=e^{-2 \omega *} \nabla_{\mu}^{(\phi)} \phi$. Clearly, (3.30) describes a conformal invariant theory. This can be identified as the Weyl gauge theory on the Weyl-Cartan geometry $Y_{4}[22[24]$.

We note that the ghost-free condition $2 a+b+c=0$ is not considered here due to the vanished kinetic term of $\phi$. It can be found that the conformal invariant theory (3.12) is a special case of the Weyl gauge invariant model with (3.21), (3.22) and the ghost-free condition $2 a+b+c=0$, implying that $2 a+b+c=0$ and $c=0$. However, this conformal invariant theory can not be identified as the gauge theory. See also the discussion for the case of $2 a+b+c=0$ in Appendix C.

We remark that the conformal invariant model in telaparallelism with the condition $2 a+b+3 c=0$ has been investigated by Maluf and Faria [12]. In their discussion, a new 
TABLE I. The model parameters $a, b$, and $c$ in the torsion scalar $T_{\mathrm{NGR}}$ with minimal and nonminimal coupled models.

\begin{tabular}{|c|c|c|c|}
\hline Model & Class & $\begin{array}{l}\text { Additional } \\
\text { condition }\end{array}$ & Reference \\
\hline \multirow{9}{*}{$\begin{array}{l}\text { Minimal coupled } \\
\frac{1}{2 \kappa} T_{\mathrm{NGR}}\end{array}$} & \multirow{2}{*}{$\begin{array}{l}2 a+b+c=0 \\
(a, b, c)=\left(\frac{1}{4}, \frac{1}{2},-1\right)\end{array}$} & - & Einstein [6] \\
\hline & & - & Cho [9] \\
\hline & \multirow{3}{*}{$\begin{array}{l}2 a+b+c=0 \\
c=-1\end{array}$} & - & Hehl et al. [10] \\
\hline & & - & Nitsch and Hehl [11] \\
\hline & & - & Hayashi and Shirafuji [7] \\
\hline & \multirow{2}{*}{$\begin{array}{l}2 a+b+c=0 \\
(a, b, c)=\left(\frac{1}{2}, 0,-1\right)\end{array}$} & \multirow{2}{*}{$\begin{array}{l}\text { Static isotropic metric } \\
\text { in Scherrer [8] }\end{array}$} & Hehl et al. [10] \\
\hline & & & Nitsch and Hehl [11] \\
\hline & $\begin{array}{l}2 a+b+c=0 \\
(a, b, c)=\left(\frac{1}{4}, \frac{1}{2},-1\right)\end{array}$ & \multirow{2}{*}{ Einstein-frame } & Geng et al. [21] \\
\hline & $\begin{array}{l}2 a+b+c=0 \\
c \leq 0\end{array}$ & & Sec. III-A \\
\hline \multirow{4}{*}{$\begin{array}{c}\text { Non-minimal coupled } \\
\text { (conformal invarience) } \\
\frac{1}{2 \kappa} \phi T_{\mathrm{NGR}}\end{array}$} & $2 a+b+3 c=0$ & $\begin{array}{l}k^{\prime} g^{\mu \nu} D_{\mu} \phi D_{\nu} \phi \\
\text { where } D_{\mu}:=\partial_{\mu}-\frac{1}{3} T_{\mu} \\
\text { with arbitrary } k^{\prime}\end{array}$ & Maluf and Faria [12] \\
\hline & $\begin{array}{l}2 a+b+c=0 \\
c=0\end{array}$ & - & Sec. III-B \\
\hline & $2 a+b+4 c=0$ & \multirow{2}{*}{$\begin{array}{l}2 a+b+c>0 \\
c \neq 0\end{array}$} & \multirow{2}{*}{ Sec. III-C } \\
\hline & $2 a+b=0$ & & \\
\hline
\end{tabular}

parameter $k^{\prime}$ for the scalar kinetic term is introduced, resulting in a four-parameters model. In our discussion, the coefficient of the kinetic term of $\phi$ is $2 a+b+c$ so that the conformal invariant theory is totally determined by three parameters only.

\section{WEAK FIELD APPROXIMATION}

If we define a canonical field $\Phi:=\sqrt{\phi / \kappa_{4}}$, the action (2.13) with matter can be written as

$$
S=S_{g}+S_{\mathrm{m}}
$$




$$
\begin{aligned}
= & \int d^{4} x\left\{e \left(\frac{1}{2} \Phi^{2} T_{\mathrm{NGR}}-\frac{a \kappa^{2} \kappa_{4}^{2}}{8} \Phi^{6} g^{\mu \rho} g^{\nu \sigma} F_{\mu \nu} F_{\rho \sigma}\right.\right. \\
& \left.\left.+(4 a+2 b+2 c) g^{\mu \nu} \partial_{\mu} \Phi \partial_{\nu} \Phi-2 c g^{\mu \nu} T_{\mu} \Phi \partial_{\nu} \Phi\right)+\kappa_{4} \lambda \Phi^{2} \mathcal{L}_{\mathrm{m}}\right\} .
\end{aligned}
$$

In the weak field approximation, the frame field is given by $e_{\mu}^{i}=\delta_{\mu}^{i}+h^{i}{ }_{\mu}$ with $\left|h^{i}{ }_{\mu}\right| \ll 1$, while the metric tensor $g_{\mu \nu}=\eta_{\mu \nu}+\gamma_{\mu \nu}$ can be read as the Minkowski background $\eta_{\mu \nu}$ with a small symmetric fluctuation $\gamma_{\mu \nu}:=2 h_{(\mu \nu)}$. The tensor $h_{\mu \nu}$ can be decomposed into the symmetric $\gamma_{\mu \nu}$ and anti-symmetric $a_{\mu \nu}:=h_{[\mu \nu]}$ parts, given by $h_{\mu \nu}=(1 / 2) \gamma_{\mu \nu}+a_{\mu \nu}$. The reciprocals of the frame fields $e_{\mu}^{i}$ are $e_{i}^{\mu}=\delta_{i}^{\mu}+f_{i}{ }^{\mu}$ with $f_{i}{ }^{\mu}=-h_{i}{ }^{\mu}$ due to the orthonormality of the frame fields. Then, the torsion tensor and vector are given by $T^{\rho}{ }_{\mu \nu}=\delta_{i}^{\rho}\left(\partial_{\mu} h^{i}{ }_{\nu}-\partial_{\nu} h^{i}{ }_{\mu}\right)+\mathcal{O}\left(h_{\mu \nu}^{2}\right)$ and $T_{\nu}=\partial_{\mu} h^{\mu}{ }_{\nu}-\partial_{\nu} h+\mathcal{O}\left(h_{\mu \nu}^{2}\right)$ at $\mathcal{O}\left(h_{\mu \nu}\right)$, respectively, where $h \equiv \delta_{i}^{\mu} h^{i}{ }_{\mu}=h^{\mu}{ }_{\mu}=(1 / 2) \gamma$ with $e=1+h+\mathcal{O}\left(h_{\mu \nu}^{2}\right)$. We expand the geometric part in the Lagrangian (4.1) and keep terms only in the lowest order:

$$
\begin{aligned}
\mathcal{L}_{g} \approx & \frac{1}{2} \Phi^{2} T_{\mathrm{NGR}}-\frac{a \kappa^{2} \kappa_{4}^{2}}{8} \Phi^{6} \eta^{\mu \rho} \eta^{\nu \sigma} F_{\mu \nu} F_{\rho \sigma} \\
& +(4 a+2 b+2 c) \eta^{\mu \nu} \partial_{\mu} \Phi \partial_{\nu} \Phi-2 c \eta^{\mu \nu} T_{\mu} \Phi \partial_{\nu} \Phi
\end{aligned}
$$

where

$$
\begin{aligned}
T_{\mathrm{NGR}}=\frac{1}{4}( & (2 a+b) \partial_{\mu} \gamma_{\nu \rho} \partial^{\mu} \gamma^{\nu \rho}-(2 a+b) \partial_{\mu} \gamma_{\nu \rho} \partial^{\rho} \gamma^{\mu \nu} \\
& \left.+c \partial^{\rho} \gamma_{\rho \mu} \partial_{\sigma} \gamma^{\sigma \mu}-2 c \partial_{\mu} \gamma \partial_{\rho} \gamma^{\rho \mu}+c \partial_{\mu} \gamma \partial^{\mu} \gamma\right) \\
& +(2 a+b) \partial_{\mu} \gamma_{\nu \rho} \partial^{\nu} a^{\mu \rho}+c \partial^{\rho} \gamma_{\rho \mu} \partial_{\sigma} a^{\sigma \mu}-c \partial_{\mu} \gamma \partial_{\rho} a^{\rho \mu} \\
& +(2 a-b) \partial_{\mu} a_{\nu \rho} \partial^{\mu} a^{\nu \rho}+(2 a-3 b) \partial_{\mu} a_{\nu \rho} \partial^{\rho} a^{\mu \nu}+c \partial^{\rho} a_{\rho \mu} \partial_{\sigma} a^{\sigma \mu}
\end{aligned}
$$

which is the well-known Fierz-Pauli Lagrangian [25] with the conditions of $(a, b, c)=$ $(1 / 4,1 / 2,-1)$ and $a_{\mu \nu}=0$. The Noether current density for the new scalar $\Phi$ becomes $J_{\mu}=(4 a+2 b+2 c) j_{\mu}$ with $j_{\mu}:=\Phi \partial_{\mu} \Phi$.

Note that the last term of $-2 c T^{\mu} \Phi \partial_{\mu} \Phi$ in (4.2) gives the scalar current $j_{\mu}$ and torsion vector $T_{\mu}$ (current-vector) interaction with the corresponding Feynman diagram shown in FIG.1. By writing $T_{\mu} \approx(1 / 2) \partial_{\rho} \gamma^{\rho}{ }_{\mu}+\partial_{\rho} a^{\rho}{ }_{\mu}-(1 / 2) \partial_{\mu} \gamma$, this term contains the anti-symmetric tensor interaction $-2 c \partial_{\rho} a^{\rho \mu} \Phi \partial_{\mu} \Phi$, reduced to $c \Phi^{2} \partial_{\mu} \partial_{\rho} a^{\rho \mu}$ by using the integration by part, which vanishes due to the symmetric property of the two derivatives. As a result, there is no contribution in the current-vector interaction from $a_{\mu \nu}$. However, the symmetric part 


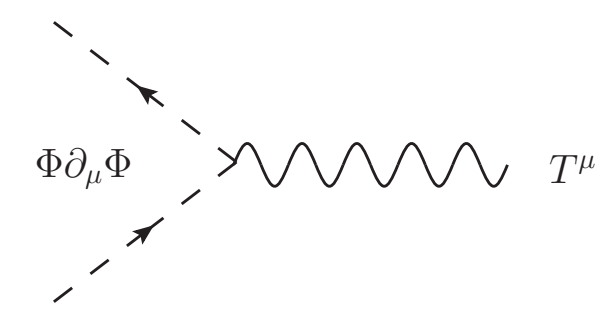

FIG. 1. The scalar current and torsion vector interaction in the four-dimensional effective Lagrangian.

cannot be eliminated even the de Donder gauge $\partial_{\rho} \gamma_{\mu}^{\rho}-(1 / 2) \partial_{\mu} \gamma=0$ is imposed unless the transversed condition $\partial_{\rho} \gamma^{\rho}{ }_{\mu}=0$ or traceless condition $\gamma=0$ is used. Clearly, it is different from GR in which the de Donder gauge would fix the degrees of freedom. On the other hand, we can take the transverse gauges for both symmetric and anti-symmetric parts, giving by $\partial_{\mu} \gamma^{\mu}{ }_{\nu}=0$ and $\partial_{\mu} a^{\mu}{ }_{\nu}=0$, respectively. By choosing $\gamma=0$, the torsion vector $T_{\mu}$ would vanish automatically, resulting in that the degrees of freedom given by the frame fields in (4.2) are the tensor modes only.

To simplify our calculation, we take that the variation of matter to be $\delta \mathcal{L}_{\mathrm{m}}=(-1 / 2) \int T^{\mu \nu} \delta h_{\mu \nu}$ with $T^{\mu \nu}:=-2 \delta \mathcal{L}_{\mathrm{m}} / \delta h_{\mu \nu}$. Due to $h_{\mu \nu}=(1 / 2) \gamma_{\mu \nu}+a_{\mu \nu}$, we define the energy-momentum tensors respect to $\gamma_{\mu \nu}$ and $a_{\mu \nu}$ are $T_{\gamma}^{\mu \nu}:=(1 / 2) T^{(\mu \nu)}=-2 \delta \mathcal{L}_{\mathrm{m}} / \delta \gamma_{\mu \nu}$ and $T_{a}^{\mu \nu}:=T^{[\mu \nu]}=$ $-2 \delta \mathcal{L}_{\mathrm{m}} / \delta a_{\mu \nu}$, respectively. The corresponding equations of motion of (4.2) for $\gamma_{\mu \nu}, a_{\mu \nu}, A_{\mu}$ and $\Phi$ are given by

$$
\begin{array}{r}
-j_{\rho}\left\{\frac{2 a+b}{2} \partial^{\rho} \gamma^{\mu \nu}-\frac{2 a+b}{4}\left(\partial^{\nu} \gamma^{\rho \mu}+\partial^{\mu} \gamma^{\rho \nu}\right)\right. \\
+\frac{c}{4}\left(\eta^{\rho \mu} \partial_{\sigma} \gamma^{\sigma \nu}+\eta^{\rho \nu} \partial_{\sigma} \gamma^{\sigma \mu}\right) \\
-\frac{c}{2}\left(\frac{1}{2} \eta^{\rho \mu} \partial^{\nu} \gamma+\frac{1}{2} \eta^{\rho \nu} \partial^{\mu} \gamma+\eta^{\mu \nu} \partial_{\sigma} \gamma^{\sigma \rho}\right) \\
+\frac{c}{2} \eta^{\mu \nu} \partial^{\rho} \gamma+\frac{2 a+b}{2}\left(\partial^{\mu} a^{\rho \nu}+\partial^{\nu} a^{\rho \mu}\right) \\
\left.+\frac{c}{2}\left(\eta^{\rho \mu} \partial_{\sigma} \alpha^{\sigma \nu}+\eta^{\rho \nu} \partial_{\sigma} \alpha^{\sigma \mu}\right)-c \eta^{\mu \nu} \partial_{\sigma} a^{\sigma \rho}\right\} \\
-\Phi^{2}\left\{\frac{2 a+b}{4} \square \gamma^{\mu \nu}-\frac{2 a+b}{8}\left(\partial_{\rho} \partial^{\nu} \gamma^{\mu \rho}+\partial_{\rho} \partial^{\mu} \gamma^{\nu \rho}\right)\right. \\
+\frac{c}{8}\left(\partial^{\mu} \partial_{\sigma} \gamma^{\sigma \nu}+\partial^{\nu} \partial_{\sigma} \gamma^{\sigma \mu}\right)-\frac{c}{4}\left(\partial^{\mu} \partial^{\nu} \gamma+\partial_{\rho} \partial_{\sigma} \gamma^{\sigma \rho} \eta^{\mu \nu}\right) \\
+\frac{c}{4} \eta^{\mu \nu} \square \gamma^{\mu}+\frac{2 a+b}{4}\left(\partial_{\rho} \partial^{\mu} a^{\rho \nu}+\partial_{\rho} \partial^{\nu} a^{\rho \mu}\right)
\end{array}
$$




$$
\begin{gathered}
\left.+\frac{c}{4}\left(\partial^{\mu} \partial_{\sigma} a^{\sigma \nu}+\partial^{\nu} \partial_{\sigma} a^{\sigma \mu}\right)\right\}+\frac{c}{2} \partial^{\mu} j^{\nu}+\frac{c}{2} \partial^{\nu} j^{\mu}-c \partial_{\rho} j^{\rho} \eta^{\mu \nu}=\frac{1}{2} \kappa_{4} \lambda \Phi^{2} T_{\gamma}^{\mu \nu}, \\
-j_{\rho}\left\{\frac{2 a+b}{2}\left(\partial^{\mu} \gamma^{\rho \nu}-\partial^{\nu} \gamma^{\rho \mu}\right)+\frac{c}{2}\left(\eta^{\rho \mu} \partial_{\sigma} \gamma^{\sigma \nu}-\eta^{\rho \nu} \partial_{\sigma} \gamma^{\sigma \mu}\right)\right. \\
-\frac{c}{2}\left(\eta^{\rho \mu} \partial^{\nu} \gamma-\eta^{\rho \nu} \partial^{\mu} \gamma\right)+2(2 a-b) \partial^{\rho} a^{\mu \nu} \\
\left.+(2 a-3 b)\left(\partial^{\mu} a^{\nu \rho}+\partial^{\nu} a^{\rho \mu}\right)+c\left(\eta^{\rho \mu} \partial_{\sigma} a^{\sigma \nu}-\eta^{\rho \nu} \partial_{\sigma} a^{\sigma \mu}\right)\right\} \\
-\Phi^{2}\left\{\frac{2 a+b}{4}\left(\partial_{\rho} \partial^{\mu} \gamma^{\rho \nu}-\partial_{\rho} \partial^{\nu} \gamma^{\rho \mu}\right)+\frac{c}{4}\left(\partial^{\mu} \partial_{\sigma} \gamma^{\sigma \nu}-\partial^{\nu} \partial_{\sigma} \gamma^{\sigma \mu}\right)\right. \\
\left.+(2 a-b) \square a^{\mu \nu}+\frac{2 a-3 b-c}{2}\left(\partial_{\rho} \partial^{\mu} a^{\nu \rho}+\partial_{\rho} \partial^{\nu} a^{\rho \mu}\right)\right\} \\
=\frac{1}{2} \kappa_{4} \lambda \Phi^{2} T_{a}^{\mu \nu}, \\
\kappa_{4} \lambda \Phi^{2} \frac{\delta \mathcal{L}_{\mathrm{m}}}{\delta A_{\mu}}+3 a \kappa^{2} \kappa_{4}^{2} \Phi^{5}\left(\partial_{\nu} \Phi\right) F^{\mu \nu}+\frac{a \kappa^{2} \kappa_{4}^{2}}{2} \Phi^{6} \partial_{\nu} F^{\mu \nu}=0 \\
\Phi T_{\mathrm{NGR}}-\frac{3 a \kappa^{2} \kappa_{4}^{2}}{4} \Phi^{5} F_{\mu \nu} F^{\mu \nu}+2 \lambda \kappa_{4} \Phi \mathcal{L}_{\mathrm{m}} \\
+\kappa_{4} \lambda \Phi^{2} \frac{\delta \mathcal{L}_{\mathrm{m}}}{\delta \Phi}-(8 a+4 b+4 c) \square \Phi+2 c \Phi \partial_{\mu} T^{\mu}=0
\end{gathered}
$$

respectively, where $\square:=\eta^{\mu \nu} \partial_{\mu} \partial_{\nu}$.

We now consider the conformal invariant model with the conditions in (3.21) and (3.22). From (4.4a) and (4.4b), along with the gauge conditions $\partial_{\mu} \gamma^{\mu \nu}=0, \partial_{\mu} a^{\mu \nu}=0$ and $\gamma=0$, we obtain

$$
\begin{gathered}
c j_{\rho}\left\{2 \partial^{\rho} \gamma^{\mu \nu}-\partial^{\mu} \gamma^{\rho \nu}-\partial^{\nu} \gamma^{\rho \mu}+2 \partial^{\mu} a^{\rho \nu}+2 \partial^{\nu} a^{\rho \mu}\right\} \\
+c \Phi^{2} \square \gamma^{\mu \nu}+\frac{c}{2} \partial^{\mu} j^{\nu}+\frac{c}{2} \partial^{\nu} j^{\mu}-c \partial_{\rho} j^{\rho} \eta^{\mu \nu}=\frac{1}{2} \kappa_{4} \lambda \Phi^{2} T_{\gamma}^{\mu \nu}, \\
j_{\rho}\left\{2 c\left(\partial^{\mu} \gamma^{\rho \nu}-\partial^{\nu} \gamma^{\rho \mu}\right)+4(b+2 c) \partial^{\rho} a^{\mu \nu}\right. \\
\left.+4(b+c)\left(\partial^{\mu} a^{\nu \rho}+\partial^{\nu} a^{\rho \mu}\right)\right\}+2(b+2 c) \Phi^{2} \square a^{\mu \nu}=\frac{1}{2} \kappa_{4} \lambda \Phi^{2} T_{a}^{\mu \nu},
\end{gathered}
$$

for $2 a+b+4 c=0$ and

$$
\begin{aligned}
\frac{c}{2} \partial^{\mu} j^{\nu}+\frac{c}{2} \partial^{\nu} j^{\mu}-c \partial_{\rho} j^{\rho} \eta^{\mu \nu} & =\frac{1}{2} \kappa_{4} \lambda \Phi^{2} T_{\gamma}^{\mu \nu}, \\
-8 a j_{\rho} f^{\rho \mu \nu}-4 a \Phi^{2} \square a^{\mu \nu} & =\frac{1}{2} \kappa_{4} \lambda \Phi^{2} T_{a}^{\mu \nu}
\end{aligned}
$$

for $2 a+b=0$, where the totally anti-symmetric tensor $f^{\rho \mu \nu}:=\partial^{\rho} a^{\mu \nu}+\partial^{\mu} a^{\nu \rho}+\partial^{\nu} a^{\rho \mu}$ is the field strength of $a^{\mu \nu}$. If we assume that the scalar field varies slowly, i.e., $\Phi \approx \Phi_{c}$ being a 
constant field, we have $j^{\mu} \approx 0$, reducing (4.5) and (4.6) to be

$$
\begin{aligned}
& \square \gamma^{\mu \nu}=\frac{\kappa_{4} \lambda}{2 c} T_{\gamma}^{\mu \nu}, \\
& \square a^{\mu \nu}=\frac{\kappa_{4} \lambda}{4(b+2 c)} T_{a}^{\mu \nu},
\end{aligned}
$$

and

$$
\begin{aligned}
0 & =T_{\gamma}^{\mu \nu}, \\
\square a^{\mu \nu} & =-\frac{\kappa_{4} \lambda}{8 a} T_{a}^{\mu \nu},
\end{aligned}
$$

respectively. The Newtonian force can be identified in (4.7a) by defining $\kappa_{4, \mathrm{eff}}:=8 \pi G_{4, \mathrm{eff}}=$ $\kappa_{4} \lambda / c$ with the effective Newtonian constant $G_{4, \text { eff }}=G_{5} / c$. It is interesting to note that only the anti-symmetric tensor $a^{\mu \nu}$ survives without the symmetric tensor field $\gamma^{\mu \nu}$ in (4.8) for the $2 a+b=0$ case, which is different from that in TEGR.

\section{CONCLUSIONS}

We have presented the five-dimensional KK theory in teleparallelism including the vector $A_{\mu}$ and scalar $\phi$ fields. The model has been generalized in a similar way as NGR with the arbitrary coefficients $a, b$ and $c$. We have decomposed the terms associated with the fifth dimension from the five-dimensional Lagrangian, as explicitly shown in Appendix A. We have concentrated on the zero mode in (2.13) and found that the coupled term between the scalar derivative and torsion vector is generated after the KK reduction, which is different from the result in GR.

We have summarized the possible choices of the coefficients $(a, b, c)$ in TABLE【for various models in the literature. To realize the Einstein-frame for the action in the four-dimensional effective action in (2.13), we have applied the conformal transformation and eliminated the gravitational non-minimal coupling terms by taking $2 a+b+c=0$ with $c \leq 0$. By choosing

$c=-1$, the Einstein-frame can be joined to the one-parameter teleparallel models [7, 10, 11]. For the conformal invariant model without the electromagnetic field, we have obtained the condition of $2 a+b=0$ with $c=0$, corresponding to a pure gravity model. Once the gauge field is introduced, we would construct the conformal gauge theory. Under the ghostfree constraints $2 a+b+c>0$ and $c \neq 0$ of (3.23), the non-minimal coupling terms of the torsion vector can be absorbed in the Kinetic term of the scalar field $\phi$ by using the modified 
covariant derivative ${ }^{*} \nabla_{\mu}^{(\phi)} \phi:=\left(\partial_{\mu}+\left(\lambda_{\phi} k / 2\right) T_{\mu}\right) \phi$ with $\lambda_{\phi}=-2$ and the torsion vector $T_{\mu}$ representing the Weyl gauge potential. In order to satisfy the conformal gauge invariance, the new condition of $2 a+b+4 c=0$ or $2 a+b=0$ without $A_{\mu}$ has been found. In such a modification, the geometry can be regarded as the Weyl-Cartan geometry $Y_{4}$, resulting in a Weyl gauge theory. We have also shown that the ghost-free conditions for the theories with the Einstein-frame and conformal invariant are $c \leq 0$ and $2 a+b+c>0$, respectively.

In terms of the redefinition of the scalar field $\Phi:=\sqrt{\phi / \kappa_{4}}$ with the mass dimension one, the non-minimal coupling of the torsion vector can be identified as the interaction of the scalar current and torsion vector. For this interaction, we have considered the weak field limit $e_{\mu}^{i}=\delta_{\mu}^{i}+h_{\mu}^{i}$ with the gravitational waves $\gamma_{\mu \nu}=g_{\mu \nu}-\eta_{\mu \nu}=2 h_{(\mu \nu)}$, in which the tensor $h_{\mu \nu}=(1 / 2) \gamma_{\mu \nu}+a_{\mu \nu}$ contains both symmetric and anti-symmetric parts. We have shown that the anti-symmetric tensor $a_{\mu \nu}$ does not contribute to the current-vector interaction. For the conformal invariant theory with $2 a+b+4 c=0$ and the slowly varying $\Phi$, the effective Newtonian constant is independent of the fifth dimensional radius $r$ of $G_{4, \text { eff }}=G_{5} / c$. Within the condition $2 a+b=0$, we have demonstrated that only the massless anti-symmetric tensor mode $a_{\mu \nu}$ can exist in the conformal invariant theory.

\section{ACKNOWLEDGMENTS}

The work was partially supported by National Center for Theoretical Sciences and Ministry of Science and Technology (MOST 104-2112-M-009-020-MY3).

\section{Appendix A: The Decomposition Terms}

We show the decompositions of the extended five-dimensional torsion scalar as follows:

$$
\begin{aligned}
\bar{T}_{\mathrm{NGR}}= & T_{\mathrm{NGR}}+4 a \kappa T_{l}^{\rho \sigma} A_{\rho}\left(\partial_{5} e_{\sigma}^{l}\right)-2 a \kappa^{2}\left(g^{\mu \rho} A_{\mu} A_{\rho}\right)\left(g^{\nu \sigma} \eta_{i l}\left(\partial_{5} e_{\nu}^{i}\right)\left(\partial_{5} e_{\sigma}^{l}\right)\right) \\
& -2 a \kappa^{2} \eta_{i l}\left(g^{\nu \rho} A_{\rho}\left(\partial_{5} e_{\nu}^{i}\right)\right)\left(g^{\mu \sigma} A_{\mu}\left(\partial_{5} e_{\sigma}^{l}\right)\right)+2 b \kappa T^{\sigma \rho}{ }_{k} A_{\rho}\left(\partial_{5} e_{\sigma}^{k}\right) \\
& -2 b \kappa T^{\rho \sigma}{ }_{k} A_{\rho}\left(\partial_{5} e_{\sigma}^{k}\right)+b \kappa^{2}\left(g^{\mu \rho} A_{\mu} A_{\rho}\right)\left(\partial_{5} e_{\nu}^{i}\right)\left(\partial_{5} e_{\sigma}^{k}\right) e_{k}^{\nu} e_{i}^{\sigma} \\
& -2 b \kappa^{2}\left(g^{\mu \sigma} A_{\mu}\left(\partial_{5} e_{\sigma}^{k}\right)\right)\left(A_{\rho} e_{i}^{\rho}\right)\left(\partial_{5} e_{\nu}^{i}\right) e_{k}^{\nu} \\
& +b \kappa^{2}\left(A_{\mu} e_{k}^{\mu}\right)\left(A_{\rho} e_{i}^{\rho}\right)\left(g^{\nu \sigma}\left(\partial_{5} e_{\nu}^{i}\right)\left(\partial_{5} e_{\sigma}^{k}\right)\right) \\
& +2 c \kappa T^{j}{ }_{j}{ }^{\sigma}\left(A_{\rho} e_{k}^{\rho}\right)\left(\partial_{5} e_{\sigma}^{k}\right)-2 c \kappa T^{j}{ }_{j}{ }^{\rho} A_{\rho} e_{k}^{\sigma}\left(\partial_{5} e_{\sigma}^{k}\right)
\end{aligned}
$$




$$
\begin{aligned}
& +c \kappa^{2}\left(A_{\mu} e_{j}^{\mu}\right)\left(A_{\rho} e_{k}^{\rho}\right)\left(g^{\nu \sigma}\left(\partial_{5} e_{\nu}^{j}\right)\left(\partial_{5} e_{\sigma}^{k}\right)\right) \\
& -2 c \kappa^{2}\left(A_{\mu} e_{j}^{\mu}\right)\left(\partial_{5} e_{\sigma}^{k}\right) e_{k}^{\sigma}\left(g^{\nu \rho} A_{\rho}\left(\partial_{5} e_{\nu}^{j}\right)\right) \\
& +c \kappa^{2}\left(g^{\mu \rho} A_{\mu} A_{\rho}\right)\left(\partial_{5} e_{\nu}^{j}\right) e_{j}^{\nu}\left(\partial_{5} e_{\sigma}^{k}\right) e_{k}^{\sigma}, \\
& \bar{T}_{i \hat{5} j} \bar{T}^{i \hat{5} j}=\frac{1}{\phi^{2}} \eta^{\hat{5} \hat{5}} \eta_{i k} g^{\mu \nu}\left(\partial_{5} e_{\mu}^{i}\right)\left(\partial_{5} e_{\nu}^{k}\right) \text {, } \\
& \bar{T}_{\hat{5} i j} \bar{T}^{\hat{5} i j}=\frac{\kappa^{2}}{4} \phi^{2} \eta_{\hat{5} \hat{5}} g^{\mu \rho} g^{\nu \sigma} F_{\mu \nu} F_{\rho \sigma}+2 \kappa^{3} \phi^{2} \eta_{\hat{5} \hat{5}} g^{\mu \sigma} g^{\nu \rho} A_{\mu}\left(\partial_{5} A_{\nu}\right) F_{\sigma \rho} \\
& +2 \kappa^{4} \phi^{2} \eta_{\hat{5} \hat{5}}\left(g^{\mu \rho} A_{\mu} A_{\rho}\right)\left(g^{\nu \sigma}\left(\partial_{5} A_{\nu}\right)\left(\partial_{5} A_{\sigma}\right)\right) \\
& -2 \kappa^{4} \phi^{2} \eta_{5 \hat{5}}\left(g^{\mu \sigma} A_{\mu}\left(\partial_{5} A_{\sigma}\right)\right)\left(g^{\nu \rho}\left(\partial_{5} A_{\nu}\right) A_{\rho}\right), \\
& \bar{T}_{i \hat{5} j} \bar{T}^{j \hat{5} i}=\frac{1}{\phi^{2}} \eta^{\hat{5} \hat{5}}\left(\partial_{5} e_{\mu}^{i}\right)\left(\partial_{5} e_{\nu}^{j}\right) e_{j}^{\mu} e_{i}^{\nu}, \\
& \bar{T}_{\hat{5} i j} \bar{T}^{j i \hat{\zeta}}=\frac{\kappa}{2} e_{j}^{\nu} g^{\mu \rho} F_{\mu \nu}\left(\partial_{5} e_{\rho}^{j}\right)-\kappa^{2}\left(A_{\mu} e_{j}^{\mu}\right)\left(g^{\nu \rho}\left(\partial_{5} A_{\nu}\right)\left(\partial_{5} e_{\rho}^{j}\right)\right) \\
& +\kappa^{2}\left(A_{\mu} g^{\mu \rho}\right)\left(\partial_{5} A_{\nu}\right)\left(\partial_{5} e_{\rho}^{j}\right) e_{j}^{\nu}, \\
& \bar{T}_{\hat{5} \hat{\zeta} \hat{5}} \bar{T}^{\hat{5} \hat{\jmath}}=\frac{1}{\phi^{2}} \eta_{\hat{5} \hat{5}} \eta^{\hat{5} \hat{5}}\left(g^{\mu \nu}\left(\partial_{\mu} \phi\right)\left(\partial_{\nu} \phi\right)\right)+\frac{2 \kappa}{\phi^{2}} \eta_{\hat{5} \hat{5}} \eta^{\hat{5} \hat{5}}\left(\partial_{5} \phi\right)\left(g^{\mu \nu}\left(\partial_{\mu} \phi\right) A_{\nu}\right) \\
& +\frac{2 \kappa}{\phi} \eta_{\hat{5} \hat{5}} \eta^{\hat{5} \hat{5}}\left(g^{\mu \nu}\left(\partial_{\mu} \phi\right)\left(\partial_{5} A_{\nu}\right)\right)+\frac{\kappa^{2}}{\phi^{2}} \eta_{\hat{5} \hat{5}} \eta^{\hat{5} \hat{5}}\left(\partial_{5} \phi\right)^{2}\left(g^{\mu \nu} A_{\mu} A_{\nu}\right) \\
& +\frac{2 \kappa^{2}}{\phi} \eta_{\hat{5} \hat{5}} \eta^{5 \hat{5}}\left(\partial_{5} \phi\right)\left(g^{\mu \nu} A_{\mu}\left(\partial_{5} A_{\nu}\right)\right)+\kappa^{2} \eta_{\hat{5} \hat{5}} \eta^{5 \hat{5}}\left(g^{\mu \nu}\left(\partial_{5} A_{\mu}\right)\left(\partial_{5} A_{\nu}\right)\right) \text {, } \\
& \bar{T}_{j i}^{j} \bar{T}_{{ }_{5}}^{i}=-\frac{1}{\phi} T^{\rho}\left(\partial_{\rho} \phi\right)-\frac{1}{\phi} T^{\rho} A_{\rho}\left(\partial_{5} \phi\right)-\kappa T^{\rho}\left(\partial_{5} A_{\rho}\right) \\
& -\frac{\kappa}{\phi}\left(A_{\mu} e_{j}^{\mu}\right)\left(g^{\nu \rho}\left(\partial_{5} e_{\nu}^{j}\right)\left(\partial_{\rho} \phi\right)\right)+\frac{\kappa}{\phi}\left(e_{j}^{\nu}\left(\partial_{5} e_{\nu}^{j}\right)\right)\left(g^{\mu \rho} A_{\mu}\left(\partial_{\rho} \phi\right)\right) \\
& -\frac{\kappa^{2}}{\phi}\left(\partial_{5} \phi\right)\left(A_{\mu} e_{j}^{\mu}\right)\left(g^{\nu \rho} A_{\rho}\left(\partial_{5} e_{\nu}^{j}\right)\right)+\frac{\kappa^{2}}{\phi^{2}}\left(\partial_{5} \phi\right)\left(\partial_{5} e_{\nu}^{j}\right) e_{j}^{\nu}\left(g^{\mu \rho} A_{\mu} A_{\rho}\right) \\
& -\kappa^{2}\left(A_{\mu} e_{j}^{\mu}\right)\left(g^{\nu \rho}\left(\partial_{5} e_{\nu}^{j}\right)\left(\partial_{5} A_{\rho}\right)\right)+\kappa^{2}\left(\partial_{5} e_{\nu}^{j}\right) e_{j}^{\nu}\left(g^{\mu \rho} A_{\mu}\left(\partial_{5} A_{\rho}\right)\right), \\
& \bar{T}_{i{ }_{i 5}}^{i} \bar{T}_{j}^{j}{ }_{j}^{\hat{5}}=\frac{1}{\phi^{2}} \eta^{\hat{5} \hat{5}}\left(\partial_{5} e_{\mu}^{i}\right) e_{i}^{\mu}\left(\partial_{5} e_{\nu}^{j}\right) e_{j}^{\nu},
\end{aligned}
$$

where $\eta_{\hat{5} \hat{5}}=\eta^{\hat{5} \hat{5}}=-1$ and $T_{\mathrm{NGR}}$ is defined in (2.4) .

\section{Appendix B: Conformal Transformation of Torsion in $D$-dimension}

The conformal transformations of the torsion tensor and vector in the $D$-dimensional spacetime are given by

$$
{ }^{(D)} \widetilde{T}^{I}{ }_{M N}=\Omega^{(D)} T^{I}{ }_{M N}+\left(\partial_{M} \Omega\right) e_{N}^{I}-e_{M}^{I}\left(\partial_{N} \Omega\right),
$$




$$
{ }^{(D)} \widetilde{T}_{M}={ }^{(D)} T_{M}+(1-D) \Omega^{-1} \partial_{M} \Omega,
$$

respectively, where $I=\hat{0}, \hat{1}, \ldots, D-1$ label the orthonormal frame and $M, N=0,1, \ldots, D-$ 1 the coordinate frame. According to (B1), the conformal transformation of the extended torsion scalar is given by

$$
\begin{aligned}
{ }^{(D)} T^{(\mathrm{ext})}= & \Omega^{2(D)} \widetilde{T}^{(\mathrm{ext})}+(4 a+2 b-(2-2 D) c) \widetilde{g}^{M N} \widetilde{T}_{M}\left(\Omega \partial_{N} \Omega\right) \\
& +\left(-2(1-D) a-(1-D) b+(1-D)^{2} c\right) \widetilde{g}^{M N} \partial_{M} \Omega \partial_{N} \Omega .
\end{aligned}
$$

\section{Appendix C: Conformal Gauge Invariance Condition}

By taking $2 a+b+c=0$ for the kinetic term of $\phi$ in (2.13), the Lagrangian is reduced to

$$
\left.\mathcal{L}_{g}\right|_{2 a+b+c=0}=e \frac{1}{2 \kappa_{4}}\left\{\phi T_{\mathrm{NGR}}-\frac{a \kappa^{2}}{4} \phi^{3} g^{\mu \rho} g^{\nu \sigma} F_{\mu \nu} F_{\rho \sigma}-2 c g^{\mu \nu} T_{\mu} \partial_{\nu} \phi\right\},
$$

while (3.5) can be read as

$$
\begin{aligned}
\left.\mathcal{L}_{g}\right|_{2 a+b+c=0}= & \widetilde{e} \frac{1}{2 \kappa_{4}}\left\{\widetilde{\phi}\left(a \widetilde{T}_{\rho \mu \nu} \widetilde{T}^{\mu \nu}+b \widetilde{T}_{\rho \mu \nu} \widetilde{T}^{\nu \mu \rho}\right)\right. \\
& \left.-6 c \widetilde{\phi} \widetilde{g}^{\mu \nu} \partial_{\mu} \omega \partial_{\nu} \omega-e^{6 \omega} \frac{a \kappa^{2}}{4} \widetilde{\phi}^{3} \widetilde{g}^{\mu \rho} \widetilde{g}^{\nu \sigma} \widetilde{F}_{\mu \nu} \widetilde{F}_{\rho \sigma}-6 c \widetilde{g}^{\mu \nu} \partial_{\mu} \omega \partial_{\nu} \widetilde{\phi}\right\} .
\end{aligned}
$$

By comparing (단 , it is apparent that (C2) without the kinetic term and the interaction of $\omega$ and $\phi$ is conformal invariant by imposing $c=0$ in the absence of the $A_{\mu}$ field. This case coincides with the discussion in Sec. IIIB, but it is not a gauge theory.

If $(2 a+b+c)>0$, we assume that $c /(2 a+b+c)=k$, i.e., $2 a+b=(c / k)-c$. The Lagrangian (2.13) can be rewritten as

$$
\begin{aligned}
\mathcal{L}_{g}= & e \frac{1}{2 \kappa_{4}}\left\{\phi\left(T_{\mathrm{NGR}}-k c g^{\mu \nu} T_{\mu} T_{\nu}\right)-\frac{a \kappa^{2}}{4} \phi^{3} g^{\mu \rho} g^{\nu \sigma} F_{\mu \nu} F_{\rho \sigma}\right. \\
& \left.+\frac{c}{k \phi}\left(g^{\mu \nu}\left(\partial_{\mu}-k T_{\mu}\right) \phi\left(\partial_{\nu}-k T_{\nu}\right) \phi\right)\right\},
\end{aligned}
$$

where $c \neq 0$. Furthermore, the coefficients in (3.5) can be read as

$$
\begin{aligned}
& 4 a+2 b+2 c=\frac{2}{k} c \\
& 14 a+7 b+c=\left(\frac{7}{k}-6\right) c,
\end{aligned}
$$




$$
\begin{aligned}
2 a+b+c & =\frac{c}{k} \\
8 a+4 b-2 c & =\left(\frac{4}{k}-6\right) c .
\end{aligned}
$$

In order to compare the Lagrangian (3.5) with (C3), we absorb the term of $\widetilde{g}^{\mu \nu} \widetilde{T}_{\mu} \partial_{\nu} \widetilde{\phi}_{\text {into }}$ the kinetic term of $\widetilde{\phi}$ in (3.5). As a result, (3.5) becomes

$$
\begin{aligned}
\mathcal{L}_{g}= & \widetilde{e} \frac{1}{2 \kappa_{4}}\left\{\widetilde{\phi} \widetilde{T}_{\mathrm{NGR}}+\frac{2}{k} c \widetilde{\phi} \widetilde{g}^{\mu \nu} \widetilde{T}_{\mu} \partial_{\nu} \omega\right. \\
& +\left(\frac{7}{k}-6\right) c \widetilde{\phi} \widetilde{g}^{\mu \nu} \partial_{\mu} \omega \partial_{\nu} \omega \\
& -e^{6 \omega} \frac{a \kappa^{2}}{4} \widetilde{\phi}^{3} \widetilde{g}^{\mu \rho} \widetilde{g}^{\nu \sigma} \widetilde{F}_{\mu \nu} \widetilde{F}_{\rho \sigma} \\
& +\frac{c}{k \widetilde{\phi}}\left(\widetilde{g}^{\mu \nu} \partial_{\mu} \widetilde{\phi} \partial_{\nu} \widetilde{\phi}-2 k \widetilde{g}^{\mu \nu} \widetilde{T}_{\mu} \widetilde{\phi} \partial_{\nu} \widetilde{\phi}\right) \\
& \left.+\left(\frac{4}{k}-6\right) c \widetilde{g}^{\mu \nu} \partial_{\mu} \omega \partial_{\nu} \widetilde{\phi}\right\} .
\end{aligned}
$$

We can use $\partial_{\nu} \widetilde{\phi}-k \widetilde{T}_{\nu} \widetilde{\phi}+k \widetilde{T}_{\nu} \widetilde{\phi}$ instead of $\partial_{\nu} \widetilde{\phi}$, leading to

$$
\begin{aligned}
\mathcal{L}_{g}= & \widetilde{e} \frac{1}{2 \kappa_{4}}\left\{\widetilde{\phi} \widetilde{T}_{\mathrm{NGR}}-k c \widetilde{\phi} \widetilde{g}^{\mu \nu} \widetilde{T}_{\mu} \widetilde{T}_{\nu}\right. \\
& +\left(4-6 k+\frac{2}{k}\right) c \widetilde{\phi} \widetilde{g}^{\mu \nu} \widetilde{T}_{\mu} \partial_{\nu} \omega \\
& +\left(\frac{7}{k}-6\right) c \widetilde{\phi} \widetilde{g}^{\mu \nu} \partial_{\mu} \omega \partial_{\nu} \omega \\
& -e^{6 \omega} \frac{a \kappa^{2}}{4} \widetilde{\phi}^{3} \widetilde{g}^{\mu \rho} \widetilde{g}^{\nu \sigma} \widetilde{F}_{\mu \nu} \widetilde{F}_{\rho \sigma} \\
& +\frac{c}{k \widetilde{\phi}}\left(\widetilde{g}^{\mu \nu}\left(\partial_{\mu}-k \widetilde{T}_{\mu}\right) \widetilde{\phi}\left(\partial_{\nu}-k \widetilde{T}_{\nu}\right) \widetilde{\phi}\right) \\
& \left.+\left(\frac{4}{k}-6\right) c \widetilde{g}^{\mu \nu} \partial_{\mu} \omega\left(\partial_{\nu}-k \widetilde{T}_{\nu}\right) \widetilde{\phi}\right\} .
\end{aligned}
$$

We also absorb the term of $\widetilde{g}^{\mu \nu} \partial_{\mu} \omega\left(\partial_{\nu}-k \widetilde{T}_{\nu}\right) \widetilde{\phi}$ into the kinetic term of $\tilde{\phi}$ as

$$
\begin{aligned}
\mathcal{L}_{g}= & \widetilde{e} \frac{1}{2 \kappa_{4}}\left\{\widetilde{\phi} \widetilde{T}_{\mathrm{NGR}}-k c \widetilde{\phi} \widetilde{g}^{\mu \nu} \widetilde{T}_{\mu} \widetilde{T}_{\nu}\right. \\
& +\left(\frac{1}{k}+2-3 k\right) 2 c \widetilde{\phi} \widetilde{g}^{\mu \nu} \widetilde{T}_{\mu} \partial_{\nu} \omega \\
& +\left(\frac{1}{k}+2-3 k\right) 3 c \widetilde{\phi} \widetilde{g}^{\mu \nu} \partial_{\mu} \omega \partial_{\nu} \omega \\
& -e^{6 \omega} \frac{a \kappa^{2}}{4} \widetilde{\phi}^{3} \widetilde{g}^{\mu \rho} \widetilde{g}^{\nu \sigma} \widetilde{F}_{\mu \nu} \widetilde{F}_{\rho \sigma}
\end{aligned}
$$




$$
\left.+\frac{c}{k \widetilde{\phi}}\left[\widetilde{g}^{\mu \nu}\left(\partial_{\mu}-k \widetilde{T}_{\mu}+(2-3 k) \partial_{\mu} \omega\right) \widetilde{\phi}\left(\partial_{\nu}-k \widetilde{T}_{\nu}+(2-3 k) \partial_{\nu} \omega\right) \widetilde{\phi}\right]\right\}
$$

so that

$$
\left(\partial_{\mu}-k \widetilde{T}_{\mu}+(2-3 k) \partial_{\mu} \omega\right) \widetilde{\phi}=e^{-2 \omega}\left(\partial_{\mu}-k T_{\mu}\right) \phi
$$

transforms as $\phi$ and gives an invariant kinetic term to be

$$
\begin{aligned}
& \widetilde{e} \frac{1}{2 \kappa_{4}} \frac{c}{k \widetilde{\phi}}\left[\widetilde{g}^{\mu \nu}\left(\partial_{\mu}-k \widetilde{T}_{\mu}+(2-3 k) \partial_{\mu} \omega\right) \widetilde{\phi}\left(\partial_{\nu}-k \widetilde{T}_{\nu}+(2-3 k) \partial_{\nu} \omega\right) \widetilde{\phi}\right] \\
= & e \frac{1}{2 \kappa_{4}} \frac{c}{k \phi}\left(g^{\mu \nu}\left(\partial_{\mu}-k T_{\mu}\right) \phi\left(\partial_{\nu}-k T_{\nu}\right) \phi\right) .
\end{aligned}
$$

In order to have the conformal gauge invariance without the vector field $A_{\mu}$ in (C7), the terms $\widetilde{\phi} \widetilde{g}^{\mu \nu} \widetilde{T}_{\mu} \partial_{\nu} \omega$ and $\widetilde{\phi} \widetilde{g}^{\mu \nu} \partial_{\mu} \omega \partial_{\nu} \omega$ in (C7) need to be absence, leading to the necessary condition

$$
\frac{1}{k}+2-3 k=0
$$

The solutions for (C10) are $k=-1 / 3$ and 1 , resulting in

$$
2 a+b=-4 c
$$

or

$$
2 a+b=0
$$

Consequently, the corresponding gauge invariant Lagrangian is

$$
\begin{aligned}
\left.\mathcal{L}_{g}\right|_{k=-\frac{1}{3}}= & \widetilde{e} \frac{1}{2 \kappa_{4}}\left\{\widetilde{\phi} \widetilde{T}_{\mathrm{NGR}}+\frac{1}{3} c \widetilde{\phi} \widetilde{g}^{\mu \nu} \widetilde{T}_{\mu} \widetilde{T}_{\nu}\right. \\
& \left.-\frac{3 c}{\widetilde{\phi}}\left[\widetilde{g}^{\mu \nu}\left(\partial_{\mu}+\frac{1}{3} \widetilde{T}_{\mu}+3 \partial_{\mu} \omega\right) \widetilde{\phi}\left(\partial_{\nu}+\frac{1}{3} \widetilde{T}_{\nu}+3 \partial_{\nu} \omega\right) \widetilde{\phi}\right]\right\}
\end{aligned}
$$

for $k=-1 / 3$ and

$$
\begin{aligned}
\left.\mathcal{L}_{g}\right|_{k=1}= & \widetilde{e} \frac{1}{2 \kappa_{4}}\left\{\widetilde{\phi}\left(a \widetilde{T}_{\rho \mu \nu} \widetilde{T}^{\rho \mu \nu}-2 a \widetilde{T}_{\rho \mu \nu} \widetilde{T}^{\nu \mu \rho}\right)\right. \\
& \left.+\frac{c}{\widetilde{\phi}}\left(\widetilde{g}^{\mu \nu}\left(\partial_{\mu}-\widetilde{T}_{\mu}-\partial_{\mu} \omega\right) \widetilde{\phi}\left(\partial_{\nu}-\widetilde{T}_{\nu}-\partial_{\nu} \omega\right) \widetilde{\phi}\right)\right\}
\end{aligned}
$$

for $k=1$.

[1] T. Kaluza, Sitzungsber. Preuss. Akad. Wiss. Berlin (Math. Phys. ) 1921, 966 (1921). 
[2] O. Klein, Z. Phys. 37, 895 (1926) [Surveys High Energ. Phys. 5, 241 (1986)].

[3] D. Bailin and A. Love, Rept. Prog. Phys. 50, 1087 (1987).

[4] J. M. Overduin and P. S. Wesson, Phys. Rept. 283, 303 (1997).

[5] A. Einstein, Sitzungsber. Preuss. Akad. Wiss., 1928(XVII), 217221, (1928).

[6] A. Einstein, Sitzungsber. Preuss. Akad. Wiss., 1929(X), 156159, (1929).

[7] K. Hayashi and T. Shirafuji, Phys. Rev. D 19, 3524 (1979) [Phys. Rev. D 24, 3312 (1982)].

[8] W. Scherrer, Z. Phys. 141 (1955) 374.

[9] Y. M. Cho, Phys. Rev. D 14, 2521 (1976).

[10] F. W. Hehl, Y. Ne'eman, J. Nitsch and P. Von der Heyde, Phys. Lett. B 78, 102 (1978).

[11] J. Nitsch and F. W. Hehl, Phys. Lett. B 90, 98 (1980).

[12] J. W. Maluf and F. F. Faria, Phys. Rev. D 85, 027502 (2012).

[13] C. Q. Geng, C. C. Lee, E. N. Saridakis and Y. P. Wu, Phys. Lett. B 704, 384 (2011); C. Q. Geng, C. C. Lee and E. N. Saridakis, JCAP 1201, 002 (2012); J. A. Gu, C. C. Lee and C. Q. Geng, Phys. Lett. B 718, 722 (2013); C. Q. Geng, J. A. Gu and C. C. Lee, Phys. Rev. D 88, 024030 (2013); J. T. Li, Y. P. Wu and C. Q. Geng, Phys. Rev. D 89, 044040 (2014).

[14] R. Ferraro and F. Fiorini, Phys. Rev. D 75, 084031 (2007).

[15] E. V. Linder, Phys. Rev. D 81, 127301 (2010) [Erratum-ibid. D 82, 109902 (2010)]; K. Bamba, C. Q. Geng and C. C. Lee, arXiv:1008.4036 [astro-ph.CO]; P. Wu and H. W. Yu, Eur. Phys. J. C 71, 1552 (2011); K. Bamba, C. Q. Geng, C. C. Lee and L. W. Luo, JCAP 1101, 021 (2011).

[16] V. C. de Andrade, L. C. T. Guillen and J. G. Pereira, Phys. Rev. D 61, 084031 (2000).

[17] A. L. Barbosa, L. C. T. Guillen and J. G. Pereira, Phys. Rev. D 66, 064028 (2002).

[18] F. Fiorini, P. A. Gonzalez and Y. Vasquez, arXiv:1304.1912 [gr-qc].

[19] K. Bamba, S. ’i. Nojiri and S. D. Odintsov, Phys. Lett. B 725, 368 (2013).

[20] C. Q. Geng, L. W. Luo and H. H. Tseng, Class. Quant. Grav. 31, 185004 (2014).

[21] C. Q. Geng, C. Lai, L. W. Luo and H. H. Tseng, Phys. Lett. B 737, 248 (2014).

[22] T. Fulton, F. Rohrlich and L. Witten, Rev. Mod. Phys. 34, 442 (1962).

[23] K. Hayashi and T. Kugo, Prog. Theor. Phys. 61, 334 (1979).

[24] M. Blagojevic, "Gravitation and gauge symmetries," Bristol, UK: IOP (2002) 522 p

[25] M. Fierz and W. Pauli, Proc. Roy. Soc. Lond. A 173, 211 (1939). 